Research Article

\title{
Unified Failure Strength Criterion for Terrace Slope Reinforcement Materials
}

\author{
Xuan Fang, ${ }^{1}$ Jie Yang $\left(\mathbb{D},{ }^{2}\right.$ Jia-Ming $\mathrm{Na}\left(\mathbb{D},{ }^{3}\right.$ and Zhen-Yuan $\mathrm{Gu} \mathbb{D}^{2}$ \\ ${ }^{1}$ School of Environment Science, Nanjing Xiaozhuang University, Nanjing 211171, China \\ ${ }^{2}$ School of Transportation and Civil Engineering, Nantong University, Nantong 226019, China \\ ${ }^{3}$ College of Civil Engineering, Nanjing Forestry University, Nanjing 210037, China \\ Correspondence should be addressed to Zhen-Yuan Gu; guzhenyuan0507@ntu.edu.cn
}

Received 7 July 2021; Revised 16 September 2021; Accepted 28 September 2021; Published 14 October 2021

Academic Editor: Nicola Baldo

Copyright (C) 2021 Xuan Fang et al. This is an open access article distributed under the Creative Commons Attribution License, which permits unrestricted use, distribution, and reproduction in any medium, provided the original work is properly cited.

This paper presents a study on the failure strength criterion of terrace slope reinforcement materials, such as lean cemented sand and gravel (LCSG) material, under a triaxial stress state. Cement content and confining pressure were selected as major factors to investigate their influence on the peak stress of terrace slope reinforcement materials based on experimental results and data from the literature. The mechanical properties of the LCSG samples, with cement contents of 60,80 , and $90 \mathrm{~kg} / \mathrm{m}^{3}$, and noncemented sand and gravel materials were tested under four confining pressure levels (namely, 300, 600, 1000, and $1500 \mathrm{kPa}$ ). The results show that the strength of LCSG material improves as the confining pressure increases. When the confining pressure exceeds $1200 \mathrm{kPa}$, the rate of increase of the strength for LCSG material and other cemented grained materials declines generally. The material strength displays a linear increase with the growth of the cement content. When the axial load rises up to a certain value, damage will occur at the particle cemented site near the shear plane, and the resistance stress generated by the cementation shows a trend of growth first and then attenuation, and concurrently, the friction between particles increases by degrees. Based on the identified strength characteristics of LCSG material under different cement contents and confining pressures, a new strength criterion that incorporates the frictional strengths and the cementing strengths is proposed for LCSG and other similar materials. The results of this work can provide an important theoretical basis for the stability calculation of terrace slopes and LCSG dams.

\section{Introduction}

In China, most regions from the south of the Qinling Mountains and to the north of the Bashan Mountains are earth-rock mountainous areas where $81 \%$ of the cultivated land is sloping land. Owing to the climatic conditions in these regions, heavy rainfall and frequent rainstorms occur for extended periods of time, resulting in severe soil erosion. Accordingly, the local government has implemented measures to transform the sloping farmlands and build level terraces to prevent soil and water loss. These level terraces are of concern to the production growth of local grains and for guaranteeing the satisfactory livelihoods of local residents. However, most terraced ridges are formed by artificial tamping that are liable to collapse considering the characteristics of soil and stone materials, the slope condition, the construction technology adopted, and unreasonable human activities. The material solidification method, which involves the addition of materials such as cement [1], high polymer [2], zeolite stabilizer [3], natural calcarenite [4], lime [5], bacteria, and other microorganisms [6] to improve the strength and stability of the gravel or soil, is used to reinforce the slopes [7]. The materials formed on the basis of the aforementioned method are essentially cemented sand and gravel, cemented coarse soil, and other cemented granular materials.

The mechanical properties of the reinforcement materials are utilized to analyze the stability of a slope theoretically, especially the strength criterion, which is used to analyze the failure criteria of the materials under various stress states and is helpful for the structural calculation of terrace slopes. Previous studies have revised the existing 
classical strength criteria and proposed several new strength criteria by carrying out triaxial tests on specific reinforcement materials. Mola-Abasi et al. [3] investigated the potential of a zeolite stabilizer known as an additive material to improve the unconfined compression strength, failure, and chemical properties of cemented sand. Coop and Atkinson [4] investigated strength properties of artificially cemented carbonate by triaxial tests at confining pressures of up to $9 \mathrm{MPa}$. With the increase of the cement content of artificially cemented carbonate, the peak strength increases which agrees well with this framework of other cemented soils. Ding et al. [8] conducted triaxial compression tests aiming at analyzing the relationship among the shear strength characteristics of solidified dredged materials, cement content, and confining pressure and then proposed a strength criterion for solidified dredged materials that accounted for the strength evolution mechanism. Li et al. [9] modified the Mohr-Coulomb (M-C) criterion through triaxial compression tests of artificially cemented sand and elaborated on the influence of the cement content. Kongsukprasert et al. [10] conducted a triaxial compression strength test on lean cemented sand and gravel (LCSG) materials and studied the effects of factors such as water content, curing agent content, dry density, curing period, and shear force on their strength. $\mathrm{Wu}$ et al. [11] analyzed the influence of curing age and confining pressure on the shear strength of LCSG materials through triaxial tests, modified the $\mathrm{M}-\mathrm{C}$ criterion, and then developed a strength criterion considering curing age. Amini and Hamidi [12] researched the effect of the cement content on the cohesion and internal friction angle values based on the M-C criterion and triaxial compression tests on LCSG. Clough et al. [13] and Wang [14] took the aggregate contents and confining pressures as the variables to determine their impact on the shear strength values of LCSG; however, no strength criterion that accounted for the aggregate content was proposed. Yang et al. [15, 16] found the influence of cement content, confining pressure, and aggregate content on the strength according to test research results and not only modified the M-C criterion but also proposed a new strength criterion for LCSG materials. Wei et al. [17] concluded from triaxial tests on LCSG materials under confining pressures of $0-2500 \mathrm{kPa}$ that the peak strength increased as the confining pressure increased, but the magnitude of the increment decreased gradually. According to the aforementioned studies, the strength criteria of LCSG materials, cement soil, and other reinforcement materials are typically directly revised according to various forms of the classical strength criteria. The strength criterion above has different forms, and most of them are obtained by directly fitting the strength and confining pressure. For the areas with abundant soil and stone materials, it is difficult to reasonably verify the slope reinforcement there based on the strength criterion of a specific cemented soil material or improved stone material given by existing references. Therefore, it is indispensable to establish a unified strength criterion.

M-C strength criterion is widely utilized because of its simple mathematical expression and clear physical meaning. To accurately reflect the decay law of slope strength, the respective reduction factors of cohesive force $c$ and friction angle $\varphi$ are applied for the M-C strength criterion. Chen et al. $[18,19]$ assumed that slope stability coefficient Fs was the weighted average of cohesion reduction coefficient and friction angle reduction coefficient, whose weight was used to represent the proportion of cohesion and friction angle, and thus proposed an improved double-strength reduction method. Various modifications to the M-C strength criterion have been proposed to reflect the characteristics of geotechnical materials [11, 20-24]. For instance, in some studies, the linear criteria were revised to nonlinear criteria according to material test results, while in others, the M-C strength criterion was modified, and binary medium models for structural soil and other similar materials were proposed. The binary medium model [23, 25-27] considers the material as a binary medium composed of a cementation element (structure) and a friction element (damaged body). The structural change in the deformation process is abstracted as structural gradual damage, which is then transformed into a damaged body. Similar to structural soil, LCSG materials have certain cemented properties. However, they are mainly provided by cemented materials such as cement and fly ash, rather than produced by structural action. Whether it is appropriate to directly simulate the strength characteristics of LCSG via binary medium modeling remains to be discussed.

Aiming at providing a theoretical basis for the stability calculation of the terrace slope of reinforcement materials such as LCSG material, the strength and failure characteristics of these cemented materials under different cement contents and confining pressures were analyzed in this study. Based on the $\mathrm{M}-\mathrm{C}$ strength criterion and binary medium theory, a new strength criterion that incorporates the frictional strengths and the cementing strengths was proposed for the LCSG material and cemented soil.

\section{Materials and Methods}

2.1. Raw Materials and Mix Ratio. Natural sand and gravel, as shown in Figure 1(a), were employed as the aggregates in the large-scale triaxial consolidated drained shear test of the LCSG material. The gradation of the aggregates is shown in Figure 2. The aggregate particle size was varying from $0.075 \mathrm{~mm}$ to $60 \mathrm{~mm}$, and the aggregate distributions are exhibited in Table 1. The sample preparation density for the aggregates was maintained at $2.29 \mathrm{~g} / \mathrm{cm}^{3}$. The LCSG materials containing cementing agents of 60,80 , and $90 \mathrm{~kg} / \mathrm{m}^{3}$ were researched, respectively, in order to accurately determine the degree of improvement of the cement content on the mechanical properties of the sand and gravel material. The cemented materials, as shown in Figure 1(a), consisted of dry cement (PO 42.5R) and secondary dry fly ash collected from power plants around Nanjing, China, respectively, which were mixed in accordance with a mass ratio of $3: 2$. The cement contents for the LCSG materials with cementing agents of 60,80 , and $90 \mathrm{~kg} / \mathrm{m}^{3}$ are 36,48 , and $54 \mathrm{~kg} / \mathrm{m}^{3}$, respectively, and the fly ash contents are 24 , 32 , and $36 \mathrm{~kg} / \mathrm{m}^{3}$, respectively. The water-binder ratio was fixed at 0.8 . 


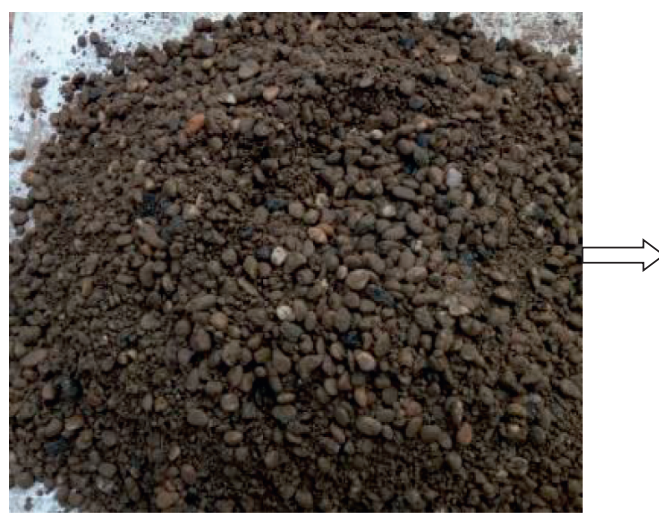

(a)

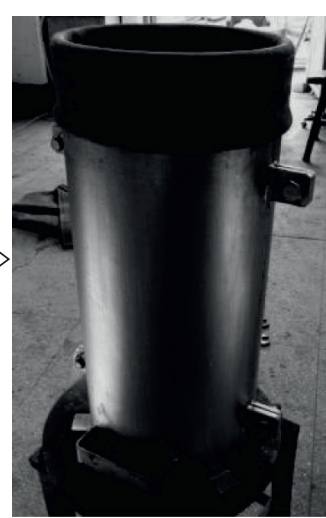

(b)

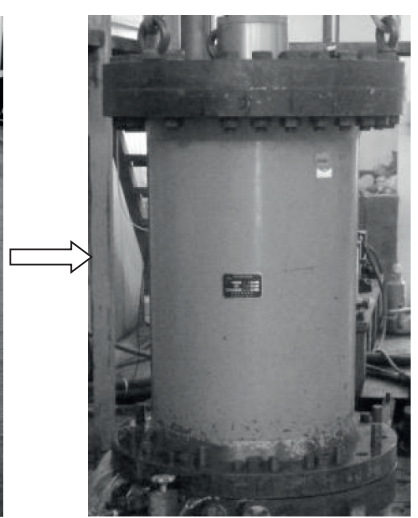

(c)

Figure 1: Raw materials and mix ratio. (a) Raw materials. (b) Cylindrical mold. (c) The static and dynamic triaxial test instrument.

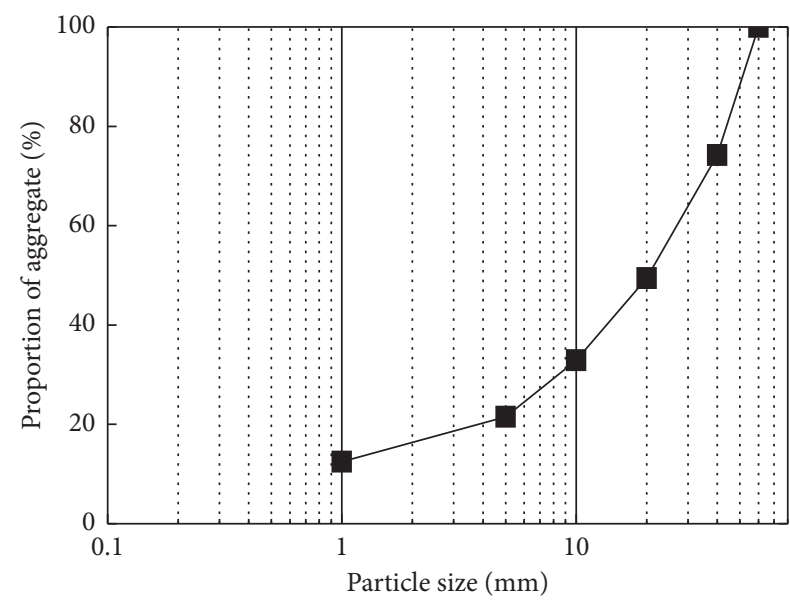

Figure 2: Gradation of the aggregates.

2.2. Test Sample Preparation. A cylindrical LCSG specimen with a diameter of $300 \mathrm{~mm}$ and a height of $700 \mathrm{~mm}$ was prepared. The specimen preparation procedure is detailed below, which has a comprehensive precision index higher than $1 \%$.

(1) The amounts of cement, fly ash, sand, gravel, and water required for each specimen were calculated according to the mix proportion requirements of cemented sand, and then these components were mixed.

(2) The mixture was divided into five layers into the cylindrical mold, as shown in Figure 1(b). Each layer was vibrated, grounded, and compacted. After the fifth mixture layer was rolled, the sample of LCSG material was formed.

(3) Two specimens were prepared for each test to improve the accuracy of the test results. The specimens were cured at $20 \pm 2^{\circ} \mathrm{C}$ for 28 days. Wu et al. [11] believed that the strength of LCSG materials had met the engineering requirements after curing for 28 days, and the strength of materials beyond 28 days had not been substantially improved. Therefore, the experimental curing age of such materials was generally set as 28 days. The specimens were fixed with a rubber sleeve to prevent them from falling or damaging the testing machine.

2.3. Test Equipment and Process. The large-scale triaxial consolidated drained shear test on LCSG was carried out using the static and dynamic triaxial test instrument as shown in Figure 1(b). The test instrument consisted of five parts, namely, the hydraulic station and digital signal element, load cell, triaxial pressure chamber, pressure/volume controller, and cylinder software. The maximum confining pressure and axial load were $4 \mathrm{MPa}$ and $1500 \mathrm{kN}$, respectively. The test was conducted according to the guidelines specified in SL237-1999 [28]. The comprehensive accuracy index of the instrument was greater than $1 \%$. In this study, the axial displacement was measured by a grating displacement sensor with an accuracy of $0.01 \mathrm{~mm}$, and the axial strain was obtained by the ratio of axial displacement to axial dimension. The volumetric strain can be calculated by the ratio of the drained volume of the specimen to its own volume.

The specimens were allowed to stand for 2 or $3 \mathrm{~h}$ prior to the triaxial tests. They were then saturated using the vacuum pumping saturation method [29] for LCSG materials, thereby ensuring that the saturation exceeded 95\%. The large-scale triaxial consolidated drained shear tests were launched under four confining pressures: 300, 600, 1000, and $1500 \mathrm{kPa}$, respectively. The reason for selecting these values of confining pressures is that they conform to the state of stress for all engineering applications of LCSG material including slope reinforcement and dam construction at present. Furthermore, it was ensured that the loading would be halted once the axial strain reached a value of $15 \%$. The specimen after the test is shown in Figure 3.

\section{Results and Discussion}

Figure 4 gives the triaxial shear test results for the noncemented sand and gravel and LCSG with cement contents of 60,80 , and $90 \mathrm{~kg} / \mathrm{m}^{3}$, respectively, under different 
TABLE 1: Details of the aggregates.

\begin{tabular}{lcccccc}
\hline Name & \multicolumn{3}{c}{ Content } \\
& $0-1(\mathrm{~mm})$ & $1-5(\mathrm{~mm})$ & $5-10(\mathrm{~mm})$ & $10-20(\mathrm{~mm})$ & $20-40(\mathrm{~mm})$ & $40-60(\mathrm{~mm})$ \\
\hline Sand and gravel $\left(\mathrm{kg} / \mathrm{m}^{3}\right)$ & 286.25 & 206.1 & 261.06 & 377.85 & 567.92 & 590.82 \\
\hline
\end{tabular}

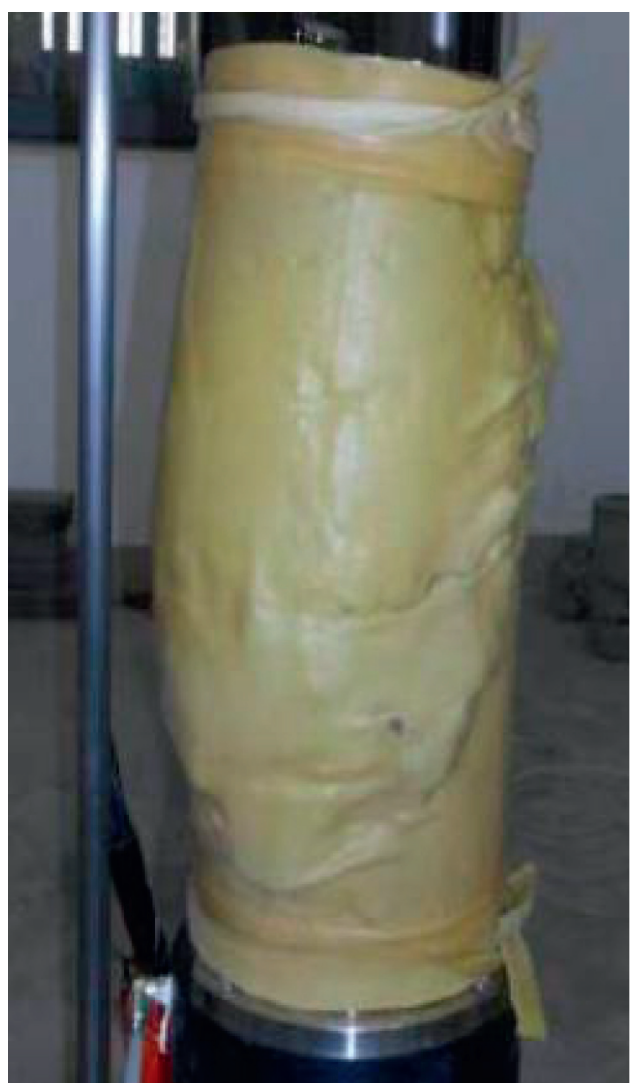

Figure 3: The specimen after the large-scale triaxial consolidated drained shear test.

confining pressures. The deviatoric stress, especially peak deviatoric stress called peak shear strength of LCSG material, grew significantly larger as the cement content increased under a fixed confining pressure. The stress-strain curves of the LCSG material under different cement contents are strain softening, which differ from the hardening curves of noncemented sand and gravel material. In contrast, the stress-strain curve of the structural loess is similar to that of the remolded loess without the strain-softening phenomenon [30]. The main reason for this difference is that the LCSG materials contain cement and fly ash, which can cement the particles and improve the strength of the sand and gravel materials, while the internal resistance of the structural loess is mainly the frictional resistance, and the damage resistance of the cement in it is negligible. To further study the strength characteristics of the LCSG material, it is necessary to understand its failure mechanism. In recent studies, Huo [31] used Particle Flow Code (PFC) software to simulate the uniaxial shear tests of the cemented coarsegrained soil, and the shear process is shown in Figure 5. Chen [32] used the multiphysics lattice discrete particle model (M-LDPM) to conduct a microscopic numerical simulation of a triaxial shear test of the cemented sand and gravel materials, the failure process of which is shown in Figure 6. Combined with the aforementioned numerical simulation results of those similar materials and the macroscopic variation characteristics of the specimen for LCSG material observed by those large-scale triaxial consolidated drained shear tests, we believe that the failure process of LCSG materials and similar materials should be shown in Figure 7. The LCSG material begins to undergo cementation damage under a certain confining pressure, and then the damaged loose particles are subjected to extrusion and friction until the LCSG material is destroyed. Macroscopically, the damaged area gradually becomes larger, forming a shear zone.

To explore the reasons of this strength enhancement, referred to the treatment method of triaxial test curves of the hardfill material at different curing ages by Wu et al. [11], the difference of the deviatoric stress between LCSG materials and noncemented sand and gravel material under the same axial strain is calculated and shown in Figure 8. At the initial stage of axial loading, the difference of the deviatoric stress of LCSG material with varying confining pressure or cemented content exists, which indicates that the cemented material in the LCSG material rapidly begins to act as a cohesive component under small strains. With the increase of axial strain, the difference of the deviatoric stress becomes greater, explaining that the cemented part of LCSG resists the load from the initial stage of loading, and the resistance increases as the loading adds. When the difference of deviatoric stress reaches the peak value of the curve, the resistance of the cemented part is the most obvious. Then, the difference of the deviatoric stress starts attenuation, reflecting that the effect of the cemented part reduces, and the deviatoric stress is mainly caused by friction. Thus, the gradual destruction of the cemented material enhances the influence of the friction between particles. The cohesive and internal frictional forces in the LCSG material develop stepwise with variations in the axial stress, the phenomenon of which resembles the properties of structural loess [30]. In addition, under the same confining pressure, the difference of the deviatoric stress corresponding to the same axial strain enlarges with the increase of the cement content. The results show that the greater the amount of cementation, the stronger the cementation resistance with the same deformation.

Combined with the deviatoric stress in Figures 4 and 8, it is seen that the differences of the peak deviatoric stress between noncemented sand and gravel and LCSG material with cement contents of 60,80 , and $90 \mathrm{~kg} / \mathrm{m}^{3}$ under a confining pressure of $300 \mathrm{kPa}$ are $1.31 \%, 1.51 \%$, and $1.29 \%$, respectively, while the corresponding differences of the peak deviatoric stress in Figure 8 are 1.28\%, 1.34\%, and 1.17\%, 


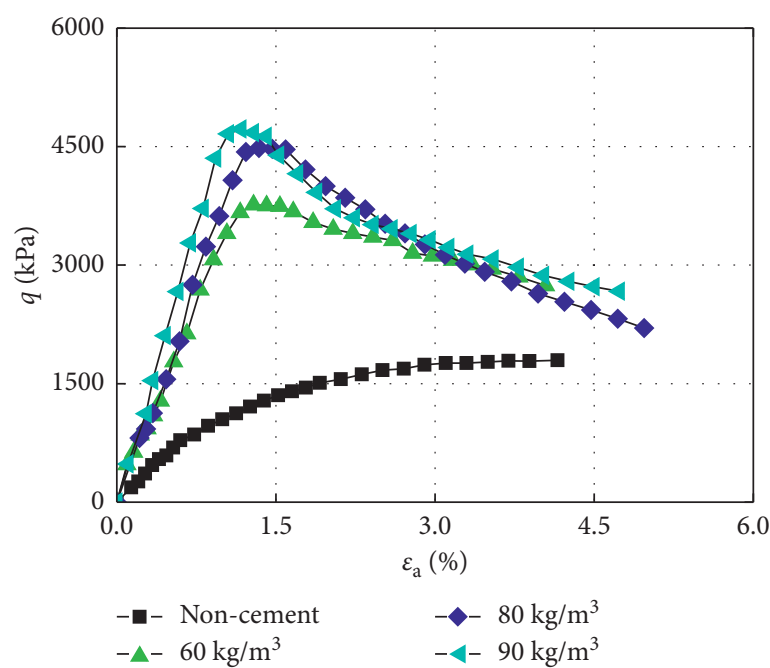

(a)

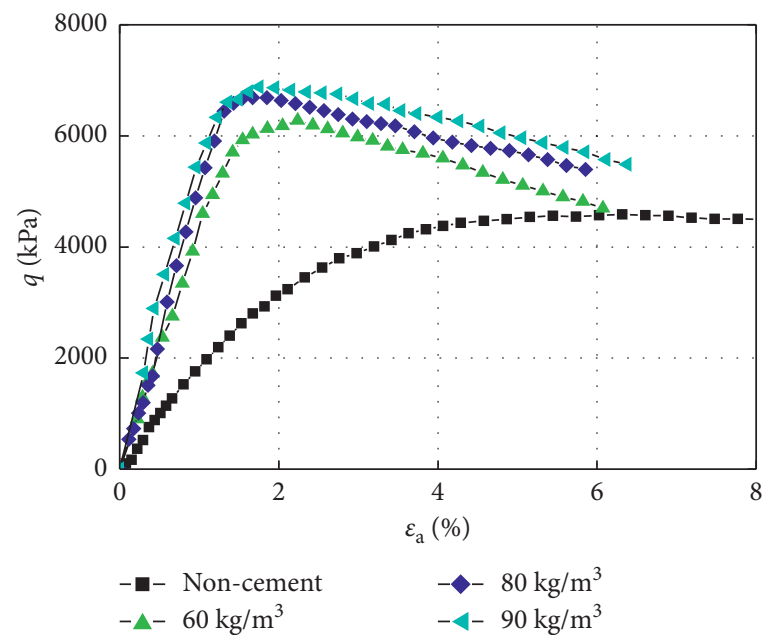

(c)

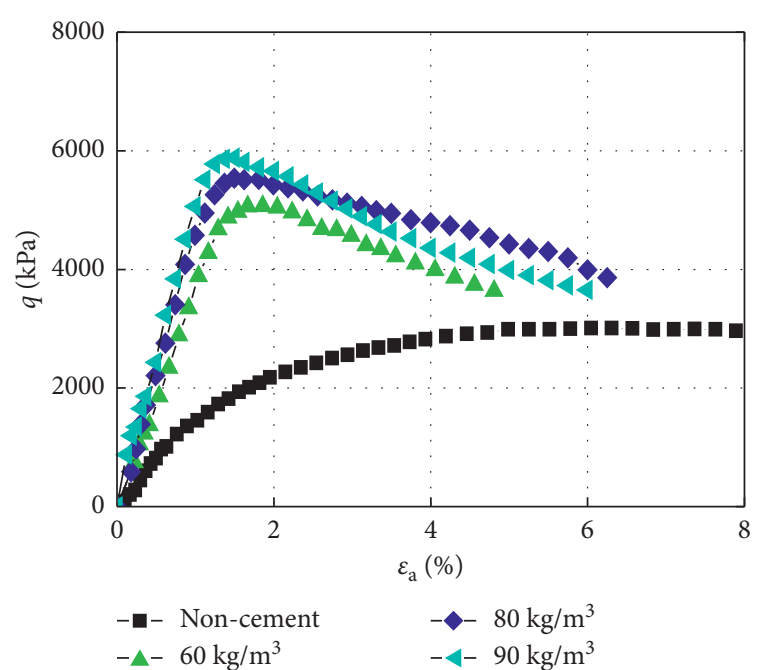

(b)

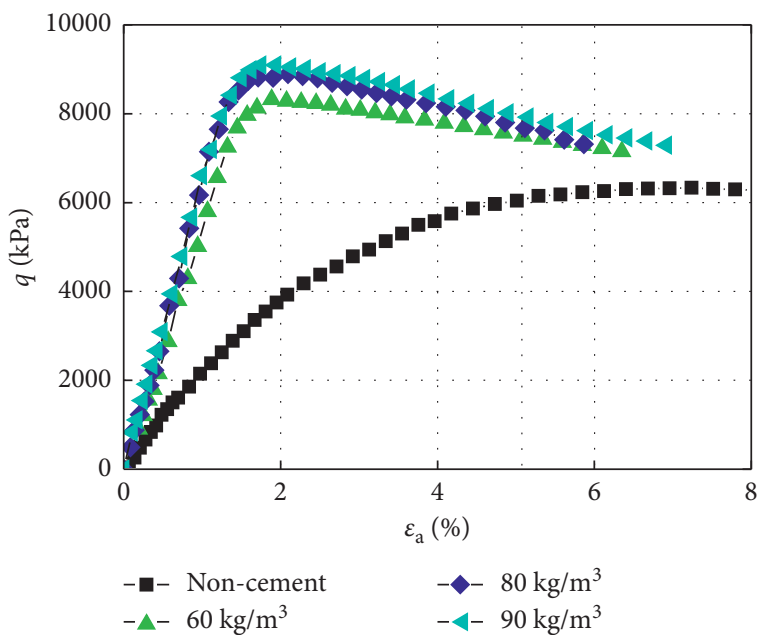

(d)

Figure 4: The stress-strain curves for different confining pressures. (a) $300 \mathrm{kPa}$. (b) $600 \mathrm{kPa}$. (c) $1000 \mathrm{kPa}$. (d) $1500 \mathrm{kPa}$.

respectively, indicating that, under a confining pressure of $300 \mathrm{kPa}$, the differences of the peak deviatoric stress of cemented sand and gravel are greater than those of the specimen under failure. The partial cementation and other partial contacts of the LCSG material particles at low pressure mainly reflect that the cementation and frictional resistance increase with the growth of the deformation. However, when the confining pressure is over $600 \mathrm{kPa}$, the differences of the peak deviatoric stress of LCSG material are smaller than those of the specimen under failure. It can be explained that the weak cementation resistance of the LCSG material attains the limit earlier, and the frictional resistance becomes the main force earlier than that of the LCSG material. Therefore, as the confining pressure augments, more particles near the shear zone can resist the external force, and the macroscopic strength value becomes greater. When the confining pressure is at a certain value, the higher the cement content of LCSG material is, the harder it is to separate the cemented particles, and the macroscopic strength value increases as well.
Figure 9 shows the relationship between the failure strength and the confining pressure of LCSG material. The strengths of the cemented and noncemented sand and gravel materials differ distinctly under the same confining pressure, illustrating that the cementation of the LCSG material significantly affects the shear strength from the beginning and contributes toward the cohesive component of shear resistance. The cemented block between the grains is compressed and damaged under the influence of the external load. Although the cemented strength of the LCSG mixture plays an important role when subjected to a low confining pressure, the residual cemented material acts as a cohesive component in the LCSG material with increasing confining pressure. The strength of the LCSG material improves nonlinearly. Wei et al. [17] increased the confining pressure to $3000 \mathrm{kPa}$ in their tests and observed that the shear strength of the LCSG mixture increased with increasing confining pressure; however, the magnitude of the increment decreased significantly, which is consistent with the results of this study. Moreover, the strength of other reinforcement materials, such as high polymer rockfill and cement 


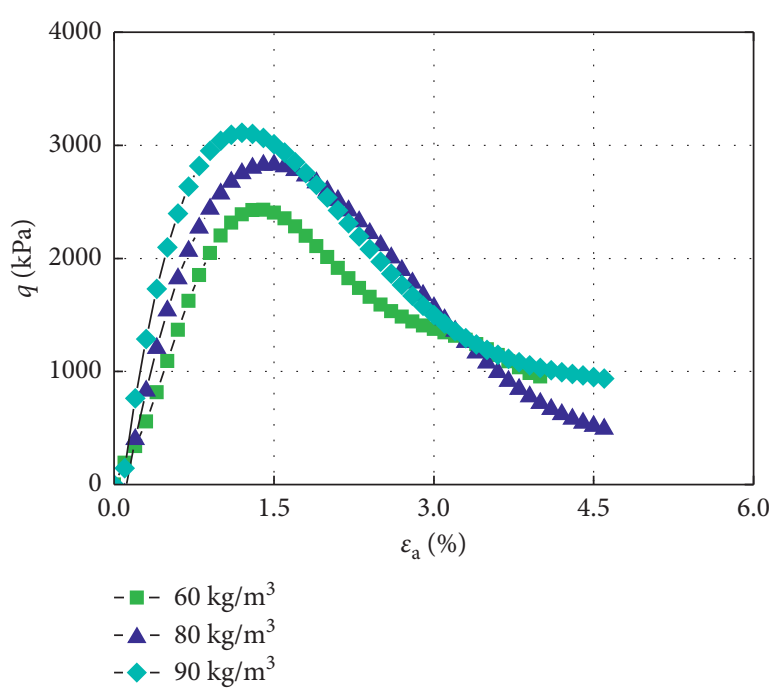

(a)

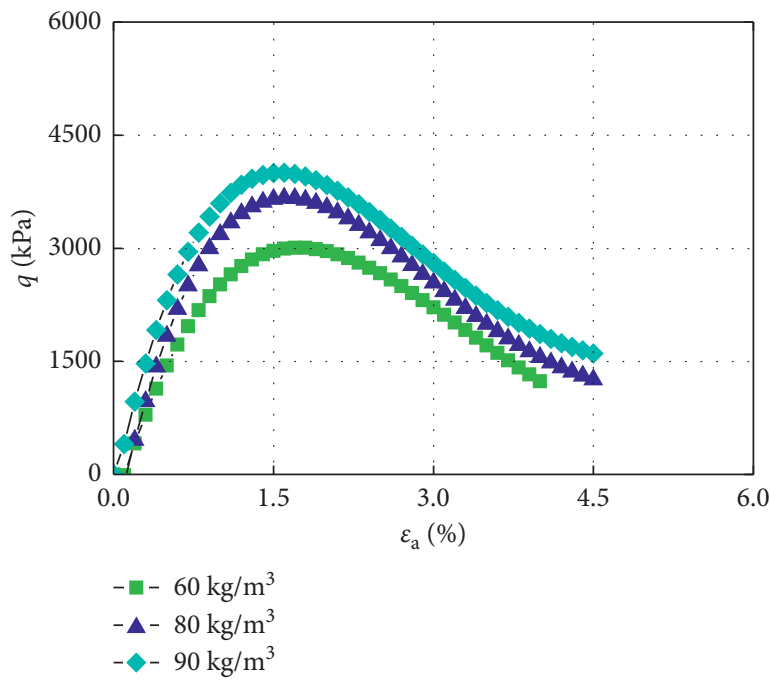

(c)

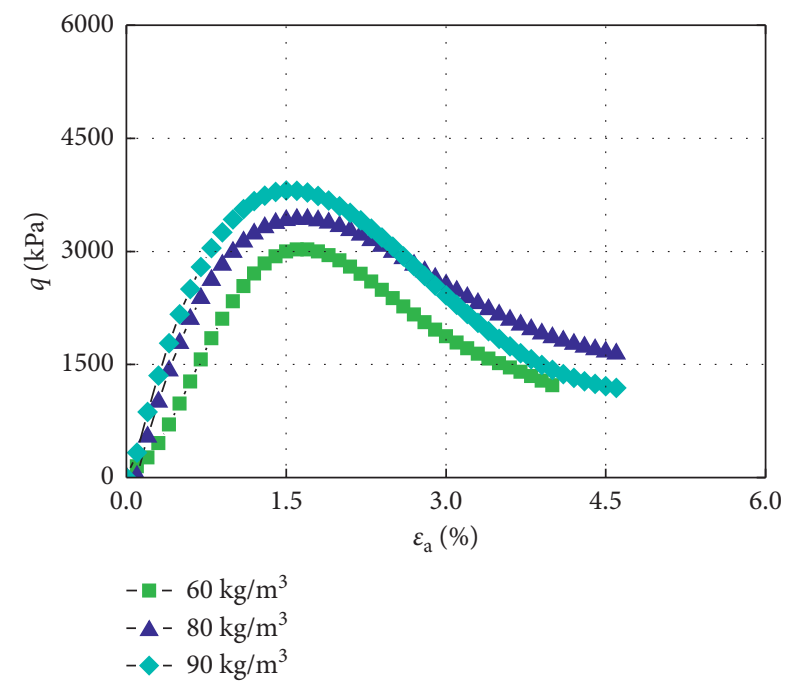

(b)

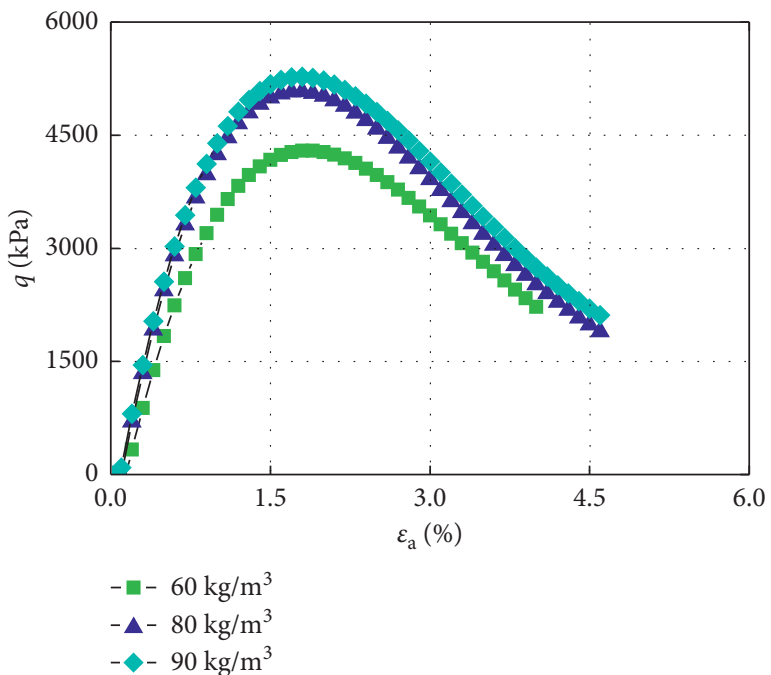

(d)

Figure 5: Difference of deviatoric stress between LCSG and noncemented sand and gravel. (a) $300 \mathrm{kPa}$. (b) $600 \mathrm{kPa}$. (c) $1000 \mathrm{kPa}$. (d) $1500 \mathrm{kPa}$.
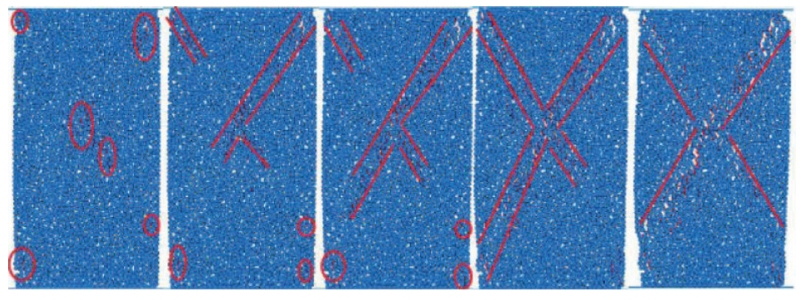

FiguRE 6: The shear failure process of cemented coarse-grained soil [31].

soil, also increases as the confining pressure accumulates, albeit accompanied by a slight reduction in the increment magnitude. Material particles are more prone to damage, and the bonding damage resistance near the failure point of these materials tends a diminution; hence, the peak strength of these materials increases nonlinearly as the confining pressure grows. The linear relationship between strength and confining pressure under the same cement content reported by Yang et al. [15] was suitable for a small range of confining pressure lower than $1200 \mathrm{kPa}$. In summary, when the confining pressure is small, a straight line can reflect the relationship between the shear strength of the LCSG material and the confining pressure. However, when the confining pressure is high, the calculated value using the linear equation is greater than the real test value, such as in the test results of Wei et al. [17]. In order to put forward a general strength criterion, we believe the nonlinear relation could reflect its strength characteristics better.

\section{Strength Criterion}

The results and discussion of LCSG material demonstrate that the shear strengths of the cemented sand and gravel mixture, the cemented soil, and other reinforcement materials are composed of resistance to cementation and friction between particles. The effect of cementation on the strength of LCSG material is much 


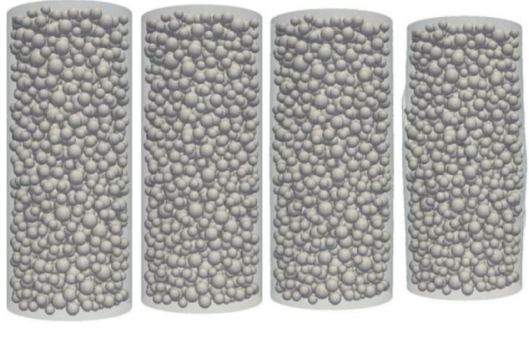

(a)
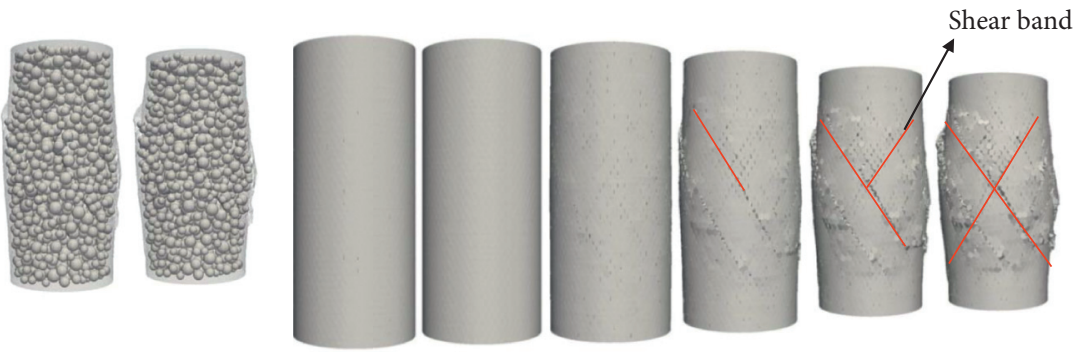

(b)

Figure 7: The shear failure process of cemented coarse-grained soil. (a) Internal particles. (b) The surface of the specimen [32].
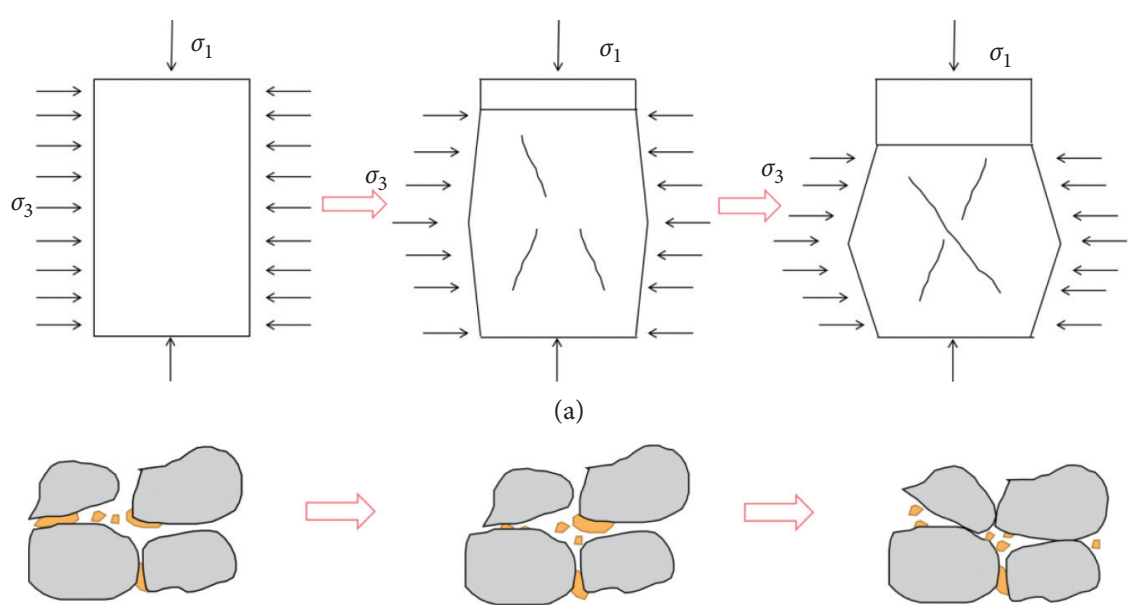

(b)

Figure 8: (a) The failure mode and (b) the failure mechanisms of the LCSG.

greater than the effect of damage resistance on the strength of loess [30]. And it is untoward to determine the shear contribution in the binary medium model. Hence, the binary medium model for structured soil is not the optimal choice to reflect the strength characteristics of LCSG material. It is essential to seek a simple and reasonable strength criterion that can describe the bonding and frictional strengths of LCSG material.

On the basis of the foregoing results as shown in Figures 4-9, the strength $q_{f}$ of the LCSG material, the cemented soil, and other reinforcement materials can be expressed as follows:

$$
q_{f}=f+A
$$

where $f$ is the stress value caused by resistance to friction and $A$ is the cementation factor which is the stress value resisting the cementation of the cement.

As indicated by Figure 9, the relationship between the strength of cemented granular materials including LCSG material and the confining pressure can be described as

$$
q_{f}=B \cdot P a\left(\frac{\sigma_{3}+P a}{P a}\right)^{n}+A .
$$

Here, $B, n$, and $A$ are dimensionless parameters; $P a$ is the standard atmospheric pressure; $B \cdot P a$ is the product of $B$ with $P a$, representing the strength of the material without cementation $(A=0)$ when the confining pressure is $0 ; n$ represents the growth index of the initial modulus.

In order to verify the accuracy of equation (3), the test results of various reinforcement materials and their corresponding calculated values are shown in Figure 10. It can be observed from the figure that, according to equation (3), the strength calculation values of LCSG material, high polymer rockfill material, and cemented soil are roughly the same as the corresponding experimental results. This indicates that the new nonlinear strength criterion is suitable for the strength prediction of different types of reinforcement materials.

The influence of parameters $A, B$, and $n$ from equation (3) on the strength was studied by utilizing the data from the LCSG material by Wei et al. [17]. The values of $A, B$, and $n$ were equal to $601.2 \mathrm{kPa}, 18.24$, and 0.51 , respectively. During the analysis of one parameter, the other parameters were kept constant. The influence of strength parameters $A, B$, and $n$ on the variations in the peak strength of the cemented sand and gravel under the confining pressure is shown in Figure 11. Increasing the value of parameter $A$ results in an augment in the predicted value of the corresponding strength, as shown in Figure 11(a); however, the form of the strength criterion remains unchanged. It is evident from Figure 11(b) that the predicted strength increases as the value of $B$ adds. With the increment of the confining 


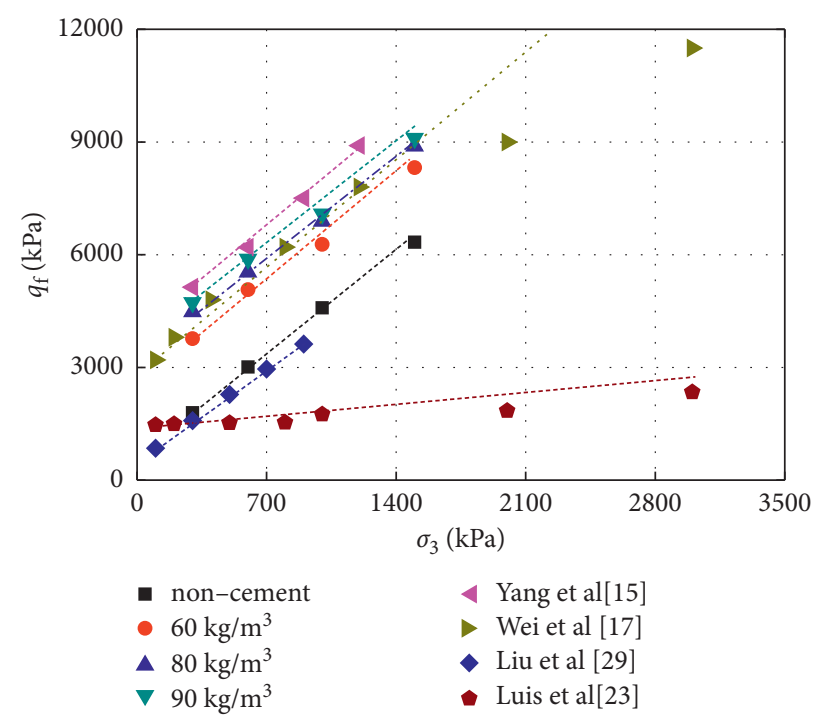

FIGURE 9: The relationship of the peak strength and confining pressure.

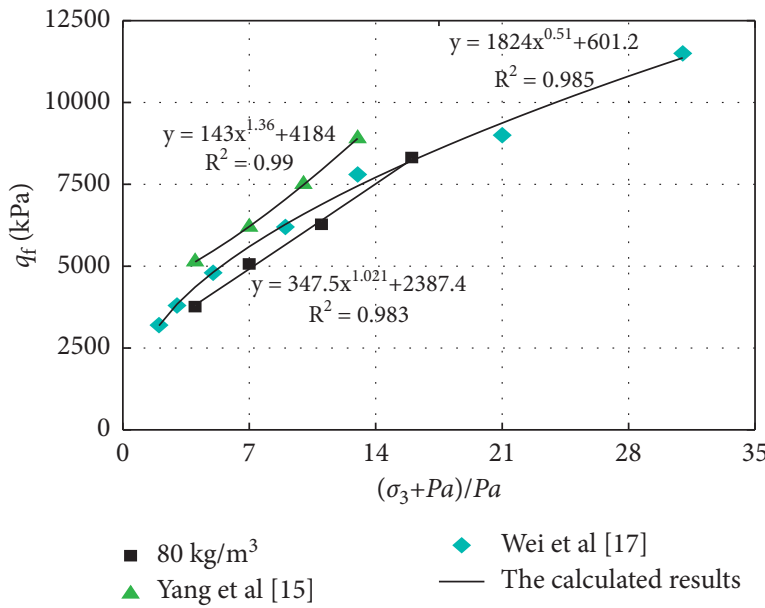

(a)

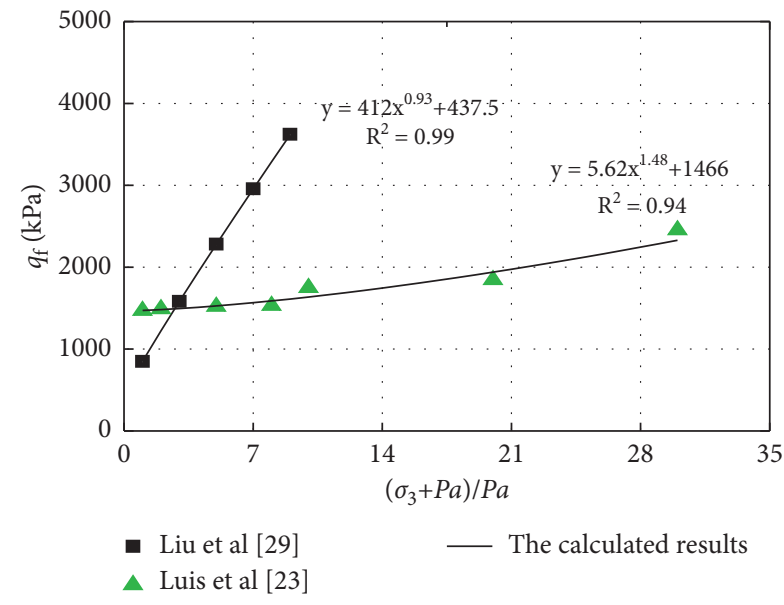

(b)

FIGURE 10: Strength criterion verification for different kinds of the reinforcement materials. (a) LCSG material. (b) Other similar reinforcement materials.

pressure, the effect of parameter $B$ on the strength of the LCSG material exceeds that of parameter $A$. Thus, the magnitude of the strength increment gradually increases. Magnifying the value of $n$ under small confining pressures leads to a slight increase in the corresponding predicted strength, as exhibited in Figure 11(c). The predicted strength value increases noticeably as the confining pressure grows. In conclusion, parameter $A$ is only related to the cementitious content, while parameters $B$ and $n$ are affected by the confining pressure, especially the high confining pressure.

Considering the effect of the cement content, $A$ in equation (2) can be rewritten as the function of cementing strength considering the influence of the cement content $C c$; when $C c$ is $0, A$ is 0 ; $B \cdot P a\left(\sigma_{3}+P a / P a\right)^{n}$ is the function of the strength due to friction.

In order to reveal the relationship between $A$ and $C c$, take LCSG material as an example. The peak strength of the noncemented sand and gravel was subtracted from the peak strengths of LCSG material with different cement contents to determine the difference of peak strength $\Delta q_{f}$ as shown in Figure 12. And the difference of peak strength $\Delta q_{f}$ of LCSG material with a specific cement content under different confining pressures is essentially the same. Therefore, the average value of the difference of peak strength $\Delta q_{f}$ can be considered as $A$ as shown in Figure 13. The relationship between $A$ and $C c$ can be expressed as

$$
A(C c)=k_{1} \frac{C c}{C c_{0}}
$$

where $k_{1}$ is the increase intensity of the relative cement content, with the unit of $\mathrm{kPa}$, and $C c_{0}$ is the reference cement content, taken as $1 \mathrm{~kg} / \mathrm{m}^{3}$.

The linear relation of equation (3) shows that the function $A$ increases linearly with the increase of the 


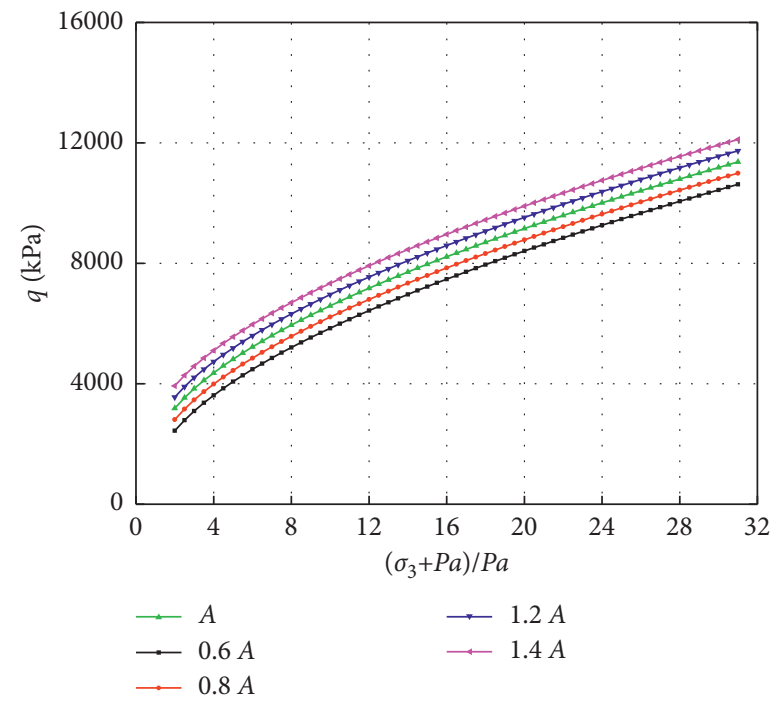

(a)

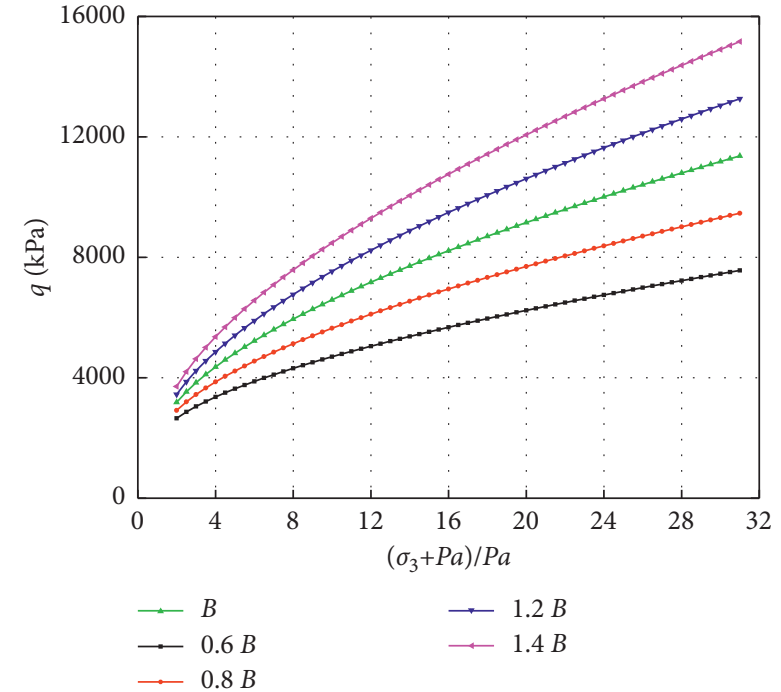

(b)

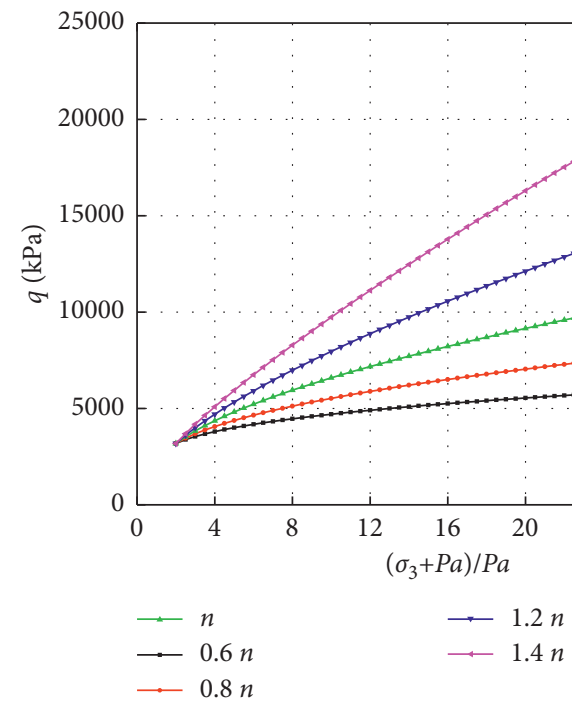

(c)

Figure 11: Influence of strength parameters on prediction results of the strength criteria. (a) Parameter $A$. (b) Parameter $B$. (c) Parameter $n$.

cement content, and only $A$ in equation (3) is affected by the cement content. Thus, the strength of LCSG material increases linearly with the increase of the cement content under the same confining pressure.

A comparison between the experimental and the predicted strength values of the LCSG material with different cement contents is illustrated in Figure 14. The predicted values of the nonlinear strength criterion, which account for the influence of the cement content, are consistent with the experimental values. This confirms that the proposed strength criterion can accurately predict the strengths of the LCSG material with different cement contents under 


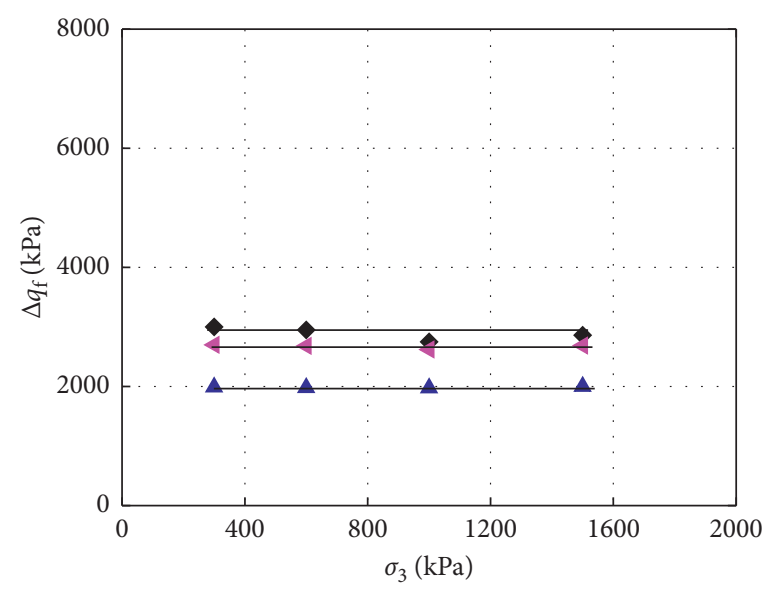

- $60 \mathrm{~kg} / \mathrm{m}^{3}$
4 $80 \mathrm{~kg} / \mathrm{m}^{3}$
- $90 \mathrm{~kg} / \mathrm{m}^{3}$

FIGURE 12: The relationship of the difference of peak strength and cement content.

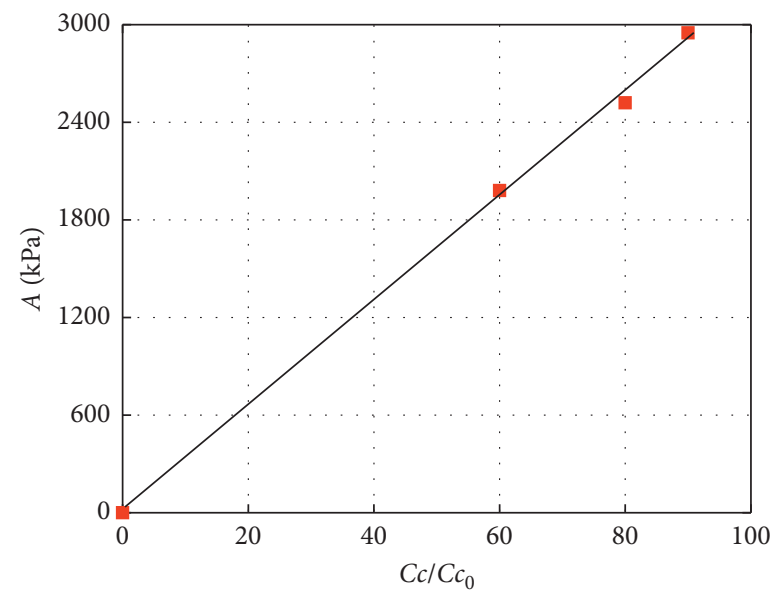

FIgURE 13: The relationship of $A$ and $C c$.

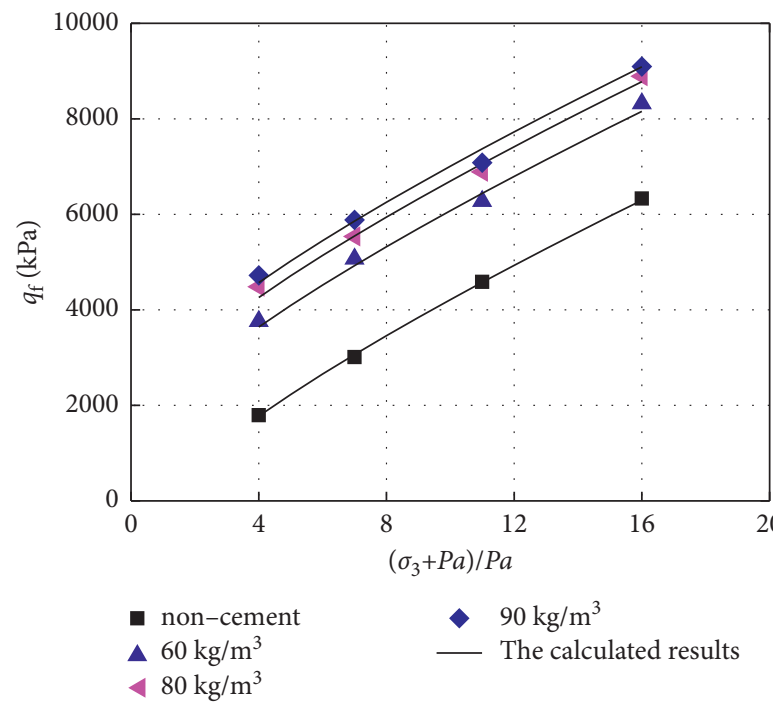

FIGURE 14: Strength criterion verification of LCSG material for different cement contents. 
varying confining pressures. In addition, it reflects that the strength increases linearly with the augment of the cement content.

\section{Conclusions}

In this study, by conducting a laboratory triaxial consolidated drained shear test of cemented granular materials such as LCSG materials and noncemented sand and gravel materials, the strength variation law of such materials under different cement contents and confining pressures was examined. The main conclusions are drawn as follows:

(1) When the stress between particles is greater than its cemented strength, failure occurs at the cementation of particles. Then, the particle friction resists the continued load, while the remaining cemented particles continue to provide cohesion against the continued load. The constantly increasing axial loading gradually damages the internal cementation point of the cylindrical specimen, resulting in the formation of an internal cross shear band.

(2) With an increase in confining pressure, failure readily occurs at the cementation of the LCSG material particles. When the stress of the LCSG material reaches the peak strength, the strength generated by the cementation first increases and then declines, and the frictional strength keeps a gradual growth.

(3) The relationship between the peak strength and the confining pressure should be nonlinear. The peak strength of the LCSG material increases linearly as the cement content adds, and the rate of increase is not affected by the confining pressure.

(4) Based on the experimental analysis and discussion results, combined with the $\mathrm{M}-\mathrm{C}$ criterion and the binary medium model, a nonlinear relation between the strength and the confining pressure of cemented granular materials including LCSG, which can reveal the cementing property and friction, is proposed in this paper. By changing the cement content, a strength criterion of cemented gravel material considering the effect of cement content and confining pressure was obtained.

These results can provide an important theoretical basis for calculations in terrace slope or other slope reinforcement engineering for the regions in rich earth and rock mixture, in addition to the existing numerical modeling of high LCSG dam structures. However, at present, those cemented coarsegrained materials including LCSG material have not been analyzed by SEM, and its detailed mesomechanism cannot be fully mastered, which will be promoted in future research of those materials by us.

\section{Data Availability}

The data used to support the findings of this study are available from the corresponding author upon request.

\section{Conflicts of Interest}

The authors declare no conflicts of interest.

\section{Acknowledgments}

This research was funded by the National Natural Science Foundation of China (Grant nos. 41871313 and 51808298), the Basic Public Welfare Research Project of Zhejiang, China (Grant no. LGF20E090002), and the Natural Science Foundation of the Higher Education Institutions of Jiangsu Province, China (Grant no. 20KJB570001).

\section{References}

[1] H. Fu, S. S. Chen, H. Q. Han, H. Ling, and X. Cai, "Experimental study on static and dynamic properties of cemented sand and gravel," Chinese Journal of Geotechnical Engineering, vol. 37, no. 2, pp. 357-362, 2015.

[2] G. Yang, X. Sun, and Y. Y. Wang, "Tests on resilient behaviour of polymer rockfill materials," Rock and Soil Mechanics, vol. 39, no. 5, pp. 1669-1674, 2018.

[3] H. Mola-Abasi, B. Kordtabar, and A. Kordnaeij, "Effect of natural zeolite and cement additive on the strength of sand," Geotechnical \& Geological Engineering, vol. 34, no. 5, pp. 1539-1551, 2016.

[4] M. R. Coop and J. H. Atkinson, "Discussion: the mechanics of cemented carbonate sands," Géotechnique, vol. 44, no. 3, pp. 533-537, 1994.

[5] A. F. Cabalar, Z. Karabash, and W. S. Mustafa, "Stabilising a clay using tyre buffings and lime," Road Materials and Pavement Design, vol. 15, no. 4, pp. 872-891, 2014.

[6] A. M. Sharaky, N. S. Mohamed, M. E. Elmashad, and N. M. Shredah, "Application of microbial biocementation to improve the physico-mechanical properties of sandy soil," Construction and Building Materials, vol. 190, pp. 861-869, 2018.

[7] A. Yildiz and F. Uysal, "Numerical modelling of Haarajoki test embankment on soft clays with and without PVDs," Geomechanics and Engineering, vol. 8, no. 5, pp. 707-726, 2015.

[8] J. W. Ding, M. L. Shi, W. Z. Liu, and Z. S. Hong, "In situ stabilization of problematic mixtures in a failed roadway subbase," Journal of Performance of Constructed Facilities, vol. 31, no. 3, pp. 707-726, 2017.

[9] D. Li, X. Liu, and X. Liu, "Experimental study on artificial cemented sand prepared with ordinary Portland cement with different contents," Materials, vol. 8, no. 7, pp. 3960-3974, 2015.

[10] L. Kongsukprasert, F. Tatsuoka, and M. Tateyama, "Several factors affecting the strength and deformation characteristics of cement-mixed gravel," Soils and Foundations, vol. 45, no. 3, pp. 107-124, 2005.

[11] M. Wu, B. Du, Y. Yao, and X. He, "An experimental study on stress-strain behavior and constitutive model of hardfill material," Science China Physics, Mechanics and Astronomy, vol. 54, no. 11, pp. 2015-2024, 2011.

[12] Y. Amini and A. Hamidi, "Triaxial shear behavior of a cementtreated sand-gravel mixture," Journal of Rock Mechanics and Geotechnical Engineering, vol. 6, no. 5, pp. 455-465, 2014.

[13] G. W. Clough, N. Sitar, R. C. Bachus, and N. S. Rad, "Cemented sands under static loading," Journal of the Geotechnical Engineering Division, vol. 107, no. 6, pp. 799-817, 1981. 
[14] Q. Wang, "Experimental study on behavior of strength a deformation of cemented coarse-grained soil," Master thesis, Dalian University of Technology, Dalian, China, 2010.

[15] J. Yang, X. Cai, Q. Pang, X.-w. Guo, Y.-1. Wu, and J.-1. Zhao, "Experimental study on the shear strength of cement-sandgravel material," Advances in Materials Science and Engineering, vol. 2018, pp. 1-11, Article ID 2531642, 2018.

[16] J. Yang, X. Cai, X. W. Guo, and J. L. Zhao, "Effect of cement content on the deformation properties of cemented sand and gravel material," Applied Sciences-Basel, vol. 9, pp. 1-16, Article ID 2369, 2019.

[17] K. M. Wei, S. S. Chen, and G. Y. Li, "Elastoplastic model for cemented coarse-grained materials and its application," Chinese Journal of Geotechnical Engineering, vol. 41, no. no5, pp. 797-805, 2019.

[18] Y. Chen, H. Lin, Y. Wang, R. Cao, C. Zhang, and Y. Zhao, "Modified double-reduction method considering strain softening and equivalent influence angle," KSCE Journal of Civil Engineering, vol. 24, no. 11, pp. 3257-3266, 2020.

[19] Y. F. Chen, H. Lin, R. H. Cao, and C. Y. Zhang, "Slope stability analysis considering different contributions of shear strength parameters," International Journal of Geomechanics, vol. 21, no. 3, pp. 1-9, Article ID 04020265, 2021.

[20] M. C. Liu, Y. F. Gao, and X. M. Huang, "Study on elastoplastic constitutive model of rockfills with nonlinear strength characteristics," Chinese Journal of Geotechnical Engineering, vol. 27, no. 3, pp. 294-298, 2005.

[21] B. A. Lingga and D. B. Apel, "Shear properties of cemented rockfills," Journal of Rock Mechanics and Geotechnical Engineering, vol. 10, no. 4, pp. 635-644, 2018.

[22] C. J. Deng, G. J He, and Y. R. Zheng, "Studies on druckerprager yield criterions based on M-C yield criterion and application in geotechnical engineering," Chinese Journal of Geotechnical Engineering, vol. 6, pp. 735-739, 2006.

[23] A. Luis, L. Deng, L. Shao, and H. Li, "Triaxial behaviour and image analysis of edmonton clay treated with cement and fly ash," Construction and Building Materials, vol. 197, pp. 208219, 2019.

[24] X. L. Li, Q. W. Li, and C. Yang, "A nonlinear failure strength criterion for rocks based on the peak value of deviatoric stress from triaxial tests," Journal of China Society, vol. 44, pp. 517-525, 2019.

[25] E. L. Liu and Z. J. Shen, "Binary medium model for structured soils," Journal of Hydraulic Engineering, vol. 36, no. 4, pp. 391-395, 2005.

[26] D. Zhang, E. Liu, X. Liu, G. Zhang, and B. Song, "A new strength criterion for frozen soils considering the influence of temperature and coarse-grained contents," Cold Regions Science and Technology, vol. 143, pp. 1-12, 2017.

[27] J. Fu, J. Wang, and W. Song, "Damage constitutive model and strength criterion of cemented paste backfill based on layered effect considerations fill based on layered effect Considerations," Journal of Materials Research and Technology, vol. 9, no. 3, pp. 6073-6084, 2020.

[28] SL237-1999, Specification of Soil Test, Ministry of Water Resources of the People's Republic of China, Beijing, China, 1999.

[29] P. Liu, H. L. Liu, Y. Xiao, and G. Yang, "Experimental study on mechanical properties of the PFA-reinforced rockfill materials," Rock and Soil Mechanics, vol. 36, no. 3, pp. 749754, 2015.

[30] B. Zhi, Y. X. Wang, B. T. Deng, and W. Liu, "Shear strength criterion for structured loess under high stress based on geo- binary medium model," Coal Engineering, vol. 50, no. 10, pp. 41-45, 2018.

[31] W. L. Huo, "Study on failure mechanism of cement sandy gravel material based on particle flow code," Master thesis, North China University of Water Conservancy and Electric Power, Zhengzhou, China, 2018.

[32] J. J. Chen, "Meso-mechanical characteristics study and multiscale analysis of cemented sand and gravel material," Doctor thesis, Hohai University, Nanjing, China, 2020. 